\title{
Esquistosomiasis vesical urinaria. Caso anatomoclínico diagnosticado en Chile
}

\author{
Ximena González', Gonzalo Méndez y David Oddó
}

\footnotetext{
'Departamento de Anatomía Patológica. Facultad de Medicina. Pontificia Universidad Católica de Chile. Santiago, Chile.

Sin conflictos de intereses. Sin financiamiento.

Recibido: 29 de agosto de 2018 Aceptado: 4 de abril de 2019

Correspondencia a: David Oddó, MD doddo@med.puc.cl
}

The

-

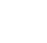

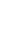


huevos parasitarios ovoideos, de 100 a $150 \mu \mathrm{m}$ de diámetro mayor, de pared gruesa ligeramente refráctil y con espolón o espina terminal, en algunos granulomas los huevos coalescieron y estaban deformados y calcificados; se concluyó el diagnóstico de cistitis granulomatosa por $S$. haematobium (Figuras 1, 2 y 3). El paciente, con este diagnóstico, fue derivado al parasitólogo clínico para su tratamiento. El paciente recibió praziquantel en dosis única de $40 \mathrm{mg}$ por $\mathrm{kg}$ de peso, evolucionando bien y sin complicaciones, transcurridos más de 10 años de evolución.

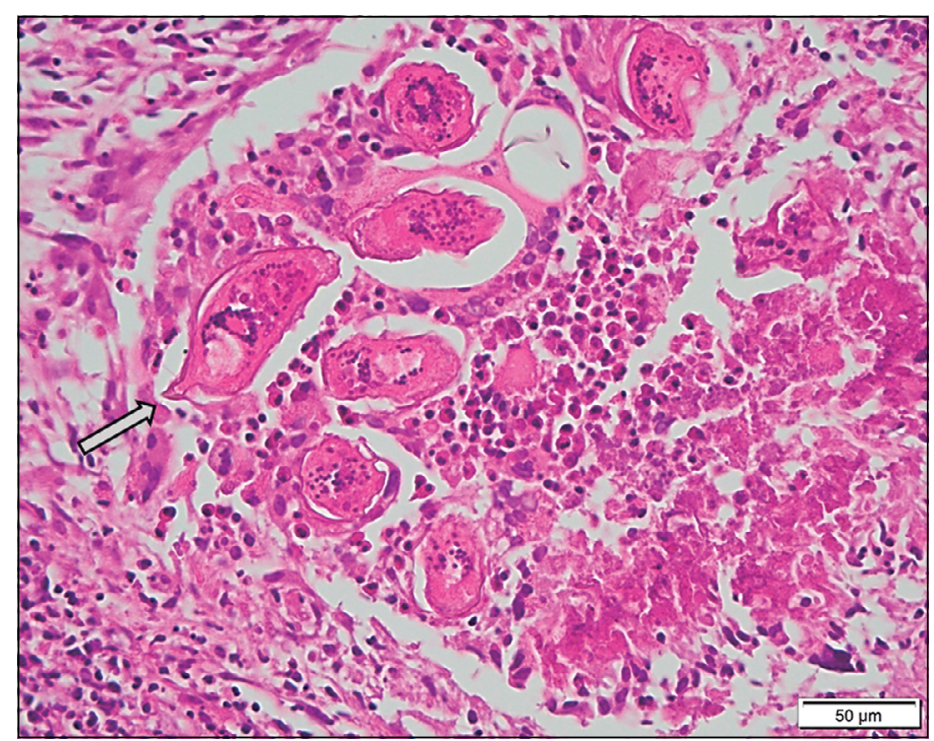

Figura 1. Esquistosomiasis vesical. Las flechas indican dos granulomas con huevos de Schistosoma haematobium (hematoxilina-eosina, X 10, aumento original).

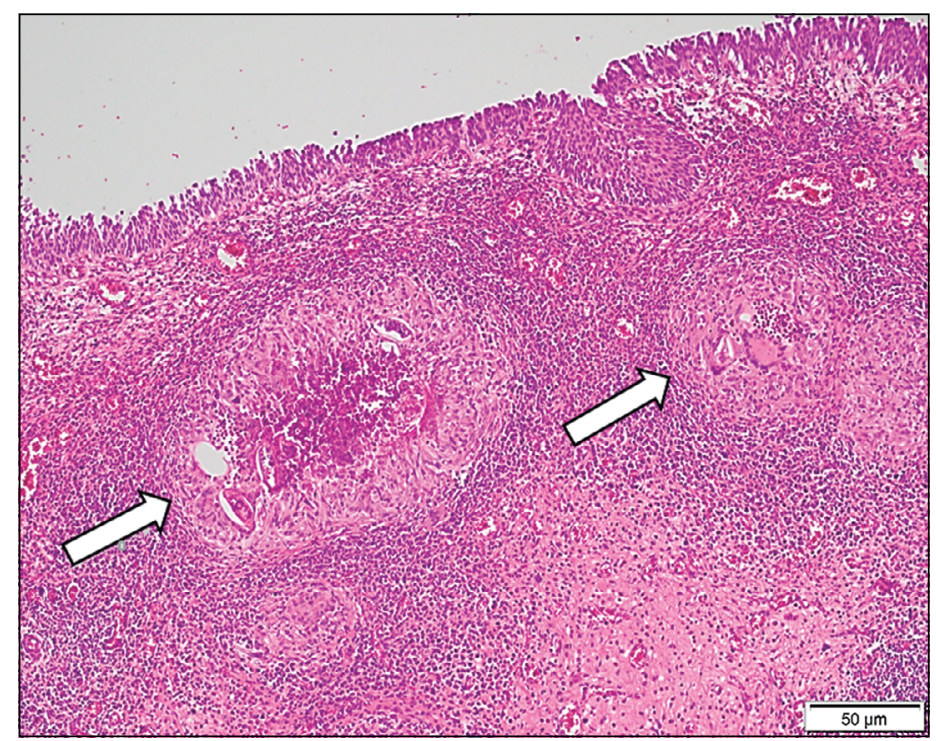

Figura 2. Esquistosomiasis vesical. Granuloma rico en leucocitos eosinófilos y varios huevos de Schistosoma haematobium. La flecha indica un huevo con espolón terminal (hematoxilina-eosina, $\times 40$, aumento original).

\section{Discusión}

Las parasitosis de las vías urinarias, a excepción de la esquistosomiasis, son infrecuentes. Sólo ocasionalmente se ha descrito la presencia de huevos o estados larvarios de helmintos en éstas; así se han publicado exclusivamente casos aislados o pequeñas series de enterobiasis, gnatostomiasis, esparginosis e hidatidosis urinarias ${ }^{14-20}$. También es muy infrecuente encontrar gusanos adultos en el tracto urinario y de forma extraordinaria se ha mencionado la presencia ectópica de Fasciola y Paragonimus $^{10}$. La presencia de larvas de moscas es otra condición excepcional $^{21}$. Por último, se agrega Vandellia cirrhosa, un pequeño pez parásito urinofílico de las aguas tropicales de América que puede introducirse en la vía urinaria baja ${ }^{22}$.

Con respecto al ciclo biológico de $S$. haematobium, el ser humano parasitado (hospedero definitivo) elimina los huevos a través de la orina; en un medio acuático propicio los huevos eclosionan como embriones ciliados (miracidios), los cuales penetran los tejidos de algunos caracoles dulceacuícolas (huésped intermediario), particularmente del género Bulinus, donde se transforman en esporoquistes que se multiplican y originan una gran cantidad de larvas (cercarias); las cercarias, al cabo de uno a dos meses, abandonan el caracol hacia el agua, muchas mueren en pocas horas, pero algunas sobreviven hasta dos días. Cuando el ser humano se baña en las aguas contaminadas, especialmente en horas soleadas y calurosas, las cercarias pueden infectarlo atravesando su piel; las larvas luego pierden su cola y se convierten en gusanos inmaduros (esquistosómulos), los que viajan a través de diversos tejidos, maduran, y se acantonan, preferentemente, en el plexo venoso perivesical urinario; finalmente los gusanos adultos copulan y tras la fecundación los huevos se depositan en la pared vesical; luego, los huevos atraviesan la pared, llegan al lumen y son excretados a través de la orina ${ }^{4,5}$.

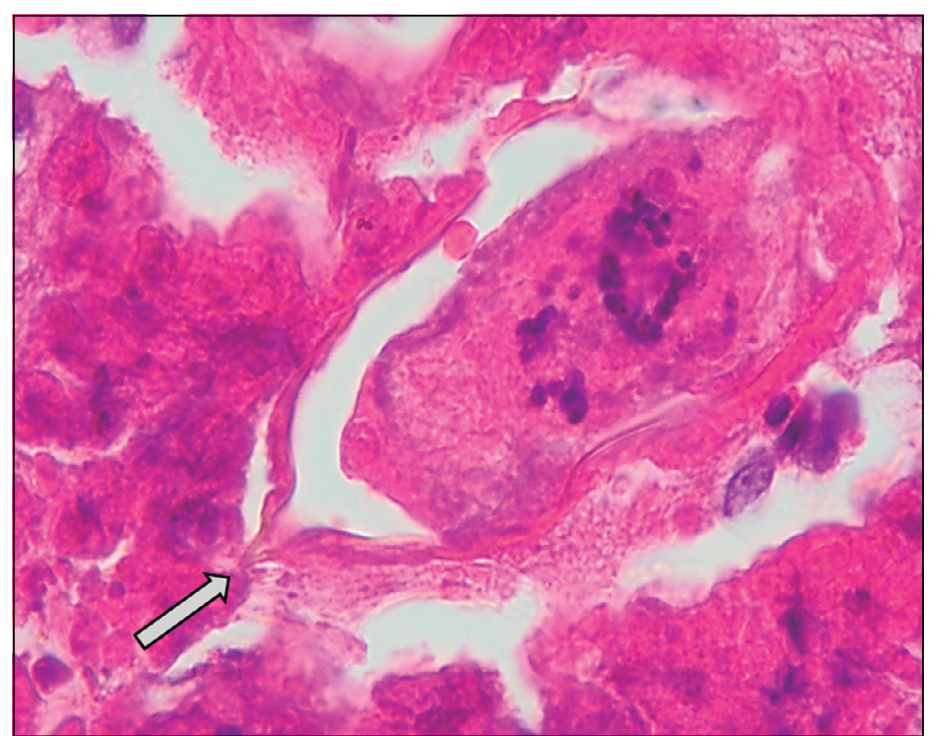

Figura 3. Esquistosomiasis vesical. Huevo de Schistosoma haematobium. La flecha indica el espolón terminal puntiagudo (hematoxilina-eosina, X 100, aumento original). 
Desde el punto de vista clínico, la esquistosomiasis vesical evoluciona en cuatro fases: una precoz, con manifestaciones cutáneas que suele durar dos a tres días; una fase invasora en la cual hay migración parasitaria y la primera ovoposición y que puede desde no presentar síntomas al síndrome de Katayama; una tercera fase o de estado, meses o años tras el contagio, caracterizada por síntomas urinarios, incluida hematuria, con eliminación de huevos en la orina; y una fase tardía de complicaciones, principalmente como consecuencia de la fibrosis cicatrizal y calcificación de la pared vesical, ureteral y uretral, que puede llevar a estenosis uretral, ureteral, hidronefrosis, atrofia e insuficiencia renal ${ }^{4,5}$. Asimismo, se considera como otra complicación tardía la aparición de carcinoma vesical, particularmente epidemoide o escamoso, aunque también de tipo transicional, posiblemente relacionados con la liberación de metabolitos carcinogénicos por parte del huevo parasitario ${ }^{4,5,22}$. En esta fase tardía, un grupo de pacientes puede desarrollar una glomerulopatía, prefentemente de tipo de mesangioproliferativa o membrano proliferativa, con hematuria y leucocituria o como un síndrome nefrótico ${ }^{4,5,24-26}$.

En el examen macroscópico de la vejiga urinaria con esquistosomiasis, se pueden encontrar tres patrones característicos: un patrón poliposo con formaciones sésiles o pediculas; un patrón con una mucosa de aspecto granular o "arenosa"; y un patrón cicatrizal y calcificado. Histológicamente, lo más llamativo es una inflamación crónica activa granulomatosa y rica en leucocitos eosinófilos, que se asienta en la mucosa y submucosa. Los granulomas contienen los huevos de S. haematobium, los que por su tamaño, su espolón o espina terminal y su ausencia de ácido-alcohol resistencia, pueden diferenciarse fácilmente de otras especies de esquistosomas. En etapas muy tardías puede haber solo una inflamación cicatrizal con calcificaciones distróficas ${ }^{10-12}$. El diagnóstico morfológico diferencial debe considerar otras cistitis granulomatosas y eosinofílicas, ya sea de tipo infeccioso o no, incluyendo la cistitis asociada al bacilo de Calmette-Guerin, tuberculosis, granulomatosis necrobiótica postquirúrgica, cistitis xantogranulomatosa, malacoplaquia, enfermedad de Wegener y otras enfermedades autoinmunes; en el caso que predomine la inflamación eosinofílica, la cistitis eosifílica asociada a un síndrome hipereosinofílico y la enfermedad relacionada a IgG4 deben considerarse como diagnóstico diferencial ${ }^{27}$.

El diagnóstico de esquistosomiasis vesical se basa en la detección de huevos parasitarios: en la orina, en la etapas activas de la enfermedad y través de un examen citológico; o en la mucosa y submucosa vesical, en fases más avanzadas, por medio de la biopsia vesical endoscópica transuretral. Dado que en el estudio rutinario del sedimento urinario, pueden no aparecer los huevos, se recomienda tomar las muestras de orina matinales, cuando la excreción de los huevos de $S$. haematobium es máxima, procedimiento que se sugiere realizar por tres días seguidos; la muestra de orina concentrada puede obtenerse por centrifugación o filtración con membranas Nucleopore de $12 \mu \mathrm{m}^{4,11}$.

El estudio con imágenes de la vejiga urinaria, particularmente la ecotomografía, es de utilidad en el estudio y seguimiento de la enfermedad ${ }^{4,5}$. Hoy en día, están disponibles pruebas inmunológicas para el diagnóstico de esquistosomiasis, y técnicas moleculares, algunas para muestras de orina, con mayor sensibilidad que el examen microscópico ${ }^{28,29}$.

El tratamiento de elección para la esquistosomiasis vesical es praziquantel en dosis única de $40 \mathrm{mg} / \mathrm{kg}$ de peso o en dos dosis de $20 \mathrm{mg} / \mathrm{kg}$ de peso del paciente, fraccionada cada $12 \mathrm{~h}$; tratamiento relatado como eficaz en todas las fases y estadios de la enfermedad ${ }^{5,30}$.

Para prevenir esta parasitosis hay que evitar el contacto con aguas contaminadas, controlar o erradicar el hospedero intermediario (caracoles), tratar oportunamente los casos, y divulgar una educación sanitaria adecuada ${ }^{30}$. Además, se están desarrollando vacunas con la finalidad de limitar la expansión de la enfermedad a nuevas zonas geográficas, rebajar la tasa de reinfección, evitar o retrasar la aparición de resistencia al tratamiento farmacológico y disminuir la incidencia de carcinoma del tracto urinario en la zonas endémicas ${ }^{2,31,32}$.

Durante los últimos años y en forma esporádica se han publicado casos aislados y una reducida serie de soldados brasileros con esquistosomiasis del tracto urinario, todos diagnosticados en países donde esta parasitosis no existe, algunos de habla castellana; en casi todos los casos, los pacientes tenían el antecedente de traslado desde territorios endémicos de infección por S. haematobium ${ }^{33-43}$.

En la revisión de los casos publicados de esquistosomiasis vesical de países sin infección endémica por $S$. haematobium, y en el caso comunicado pero no publicado en Chile $^{8}$, la presentación, estudio y tratamiento de los casos es muy similar. Los pacientes siempre fueron inmigrantes o viajeros de zonas endémicas de esquistosomiasis, que presentaron hematuria macroscópica; en la mayoría de los casos la hipótesis diagnóstica de esquistosomiasis vesical se planteó en una segunda instancia; y el diagnóstico definitivo se concretó por medio del análisis especial de orina y por la biopsia endoscópica transuretral de la vejiga, en cerca de partes iguales; y casi todos los pacientes fueron tratados con praziquantel con aparente éxito ${ }^{8,32,33,35,36,38,39-42}$.

El aumento de viajes, desde nuestro país a zonas endémicas de esta parasitosis, y el incremento de la inmigración hacia Chile en los últimos años, nos debe poner en alerta sobre la necesidad de considerar la esquistosomiasis vesical como diagnóstico diferencial en un síndrome miccional con hematuria. 


\section{Resumen}

La esquistomiasis urinaria es producida por Schistosoma haematobium. Es una enfermedad endémica en muchas regiones del mundo, no existente en Chile. Se presenta el caso de un hombre joven que viajó a Malawi, en África meridional, y que a su regreso al país, años después, presentó un síndrome miccional con hematuria macroscópica. La biopsia de vejiga mostró una cistitis granulomatosa y eosinofilica con huevos de Schistosoma haematobium.

\section{Referencias bibliográficas}

1.- Kehinde E O, Anim J T, Hira P R. Parasites of urological importance. Urol Int 2008; 81: 1-13.

2.- Ballesta Martínez B, Rodríguez Talavera J, Amador Robayna A, Carrión Valencia A, Orribo Morales N, García García L, et al. Parasitic hematuria: six cases in a row in a single centre in Spain. Urol Int 2017. doi: 10.1159/000479334.

3.- Prata A, Neghme A. Esquistosomiasis. Capítulo 36. En: Atias A Neghme E editores. Parasitología clínica. Santiago-Chile: Publicaciones Técnicas Mediterráneo; 1985. p 290-6.

4.- Gray D J, Ross A G, Li Y S, McManus D P. Diagnosis and management of schistosomiasis. BMJ 2011; 342: d2651. doi: 10.1136/bmj. d2651.

5.- Barsoum R S, Esmat G, El-Baz T. Human schistosomiasis: clinical perspective: review. J Adv Res 2013; 4: 433-44. doi: 10.1016/j. jare.2013.01.005.

6.- Daiber A. Esquistosomiasis por Schistosoma mansoni. Rev Med Chile 1974; 102: 697.

7.- Oddó D, Jarpa A, Thompson L. Colitis por Schistosoma mansoni. Rev Med Chile 1986; 114: 973-977.

8.- Aylwin M, Araya A. Infección urinaria por Schistosoma haematobium: caso clínico, en XI Jornadas Científicas, Instituto de Salud Pública de Chile, Santiago, 2013, p. 60.

9.- Pérez Sánchez YR. Esquistosomiasis urogenital. Aportación de un caso clínico. Rev Chil Urol 2018; 83 (2): 21-24.

10.- Gutiérrez Y. Trematodes of the blood vessel - Schistosomiasis. In: Gutiérrez Y, editor. Diagnostic pathology of parasitic infections with clinical correlations. New York: Oxford University Press; 2000. P 545-576.

11.- Cheever A W, Neafie R C. Schistosomiasis. Volume I Helminthiases, Chapter 2. In: Meyers WM editor. Pathology of infectious diseases. Washington: Armed Forces of Pathology; 2000. p 23-48.

12.- Von Lichtenberg F. Schistosomiasis. Volume 2 chapter 175. In: Connor AND, Chandler FW, Schwartz DA, Manz HJ, Lack EE, editors. Pathology of infectious diseases. Stamford, Connecticut: Appleton \& Lange; 1997. p. 153751.

13.- Akinwale O P, Hock T T, Chiang-Kwung F, Zheng Q, Haimo S, Ezeh C, et al.
Differentiating Schistosoma haematobium from Schistosoma magrebowiei and other closely related schistosomas by polymerase chain reaction amplification of a species specific mitochondrial gene. Trop Parasitol 2014; 4 : 38-42. doi: 10.4103/2229-5070.129163.

14.- Zahariou A, Karamouti M, Papaioannou P. Enterobius vermicularis in the male urinary tract: a case report. J Med Case Rep 2007; 1 : 137.

15.- Sunirmal C, Kumar B, Kumar Pal D. Enterobius vermicularis infestation of urinary tract leading recurrent urinary tract infection. Trop Parasitol 2017; 7: 119-21. doi: 10.4103/ tp.TP_22_17.

16.- Nitidandhaprabhas P, Sirikarna A, Harnsomburana K, Thepsitthar P. Human urinary gnathostomisis: a case report from Thailand. Am J Trop Med Hyg 1975; 24 : 49-51.

17.- Horohoe JJ, Ritterson AL, Chessin LN. Urinary gnathostomiasis. JAMA 1984; 251: 255-6.

18.- Trupti B, Shirish N, Maneesha P, Santosh A. An unsual case of urinary sparganosis in the Indian subcontinent. Indian J Urol 2018; 34: 158-60. doi: 10.4103/iju.IJU_273_17.

19.- Zargar-Shoshtari M, Shadpour P, Robat-Moradi $\mathrm{N}$, Moslemi M. Hydatid cyst of the urinary tract. Urol J (Tehran) 2007; 4: 41-5.

20.- Ganie F A, Dar O H, Kaleem A, Hassan S, Gani M. Hydatid cyst of urinary bladder. Indian J Nephrol 2013; 23: 462-3. doi: 10.4103/09714065.120348.

21.- Rasti S, Dehghani R, Naeimi Khaledi H, Takhtfiroozeh S M, Chimehi E. Uncommon human urinary tract myasis due Psychoda $s p$. Larvae, Kashan, Iran: a case report. Iran J Parasitol 2016; 11: 417-21.

22.- Arango Toro OJ, Arbeláez Arango S, Franco Miranda E. La Vandellia cirrhosa, un parásito urológico poco conocido. Actas Urol Esp 2001; 25: 325-6.

23.- Rambau P F, Chalya P L, Jackson K. Schistosomiasis and urinary bladder cancer in North Western Tanzania: a retrospective review of 185 patients. Infect Agents Cancer 2013; 8: 19. doi: 10.1186/1750-9378-8-19.

24.- Barsoum R S. Schistosomal glomerulopathies. Kidney Int 1993; 44: 1-12.

25.- Seck S M, Sarr M L, Dial M C, Ka E F. Schistosoma hematobium associated glomerulopathy. Indian J Nephrol 2011; 21: 201-3. doi: 10.4103/0971-4065.78076.

26.- Bezerra da Silva G, Bezerra Duarte D,
Guardao Barros E J, De Francesco Daher E. Schistosomiasis associated kidney disease: a review. Asian Pac J Trop Dis 2013; 3: 79-84. doi: 10.1016/S2222-1808(13)60018-3.

27.- Kojima K, Maeda J, Mikami S, Yamagishi H, Ide H, Hattori S, et al. Eosinophilic cystitis presented as manifestation o hypereosinophilic syndrome: a case report and review of the literature. Nephron Extra 2013; 3: 30-5. doi: $10.1159 / 000346713$.

28.- Aryeetey Y A, Eissien-Baidoo S, Larbi IA, Ahmed K, Amoah A S, Obeng B B, et al. Molecular diagnosis of Schistosoma infections in urine samples of school in Ghana. Am J Trop Med Hyg 2013; 88: 1028-31. doi: 10.4269/ ajtmh.12-0571.

29.- Weinfeng G, Joseph W M, Zhisheng D, Yumin Z, Wei H. Advances in diagnosis of schistosomiasis. Microbiol Curr Res 2018; 2: 14-9.

30.- Apt W. Esquistosomiasis por Schistosoma haematobium. Capítulo 65. En: Apt W editor. Parasitología humana. México: McGraw Hill Interamericana Editores SA; 2013. p 496-7.

31.- Pearson M S, Becker L, Driguez P, Young N D, Gaze S, Mendes T, et al. Of monkeys and men: immunomic profiling of sera from humans and non-human primates resistant to schistosomiasis reveals novel potential vaccine candidates. Front Immunol 2015; 6: 213. doi: 10.3389/fimmu.2015.00213.

32.- Molehin A J, Rojo J U, Siddiqui S Z, Gray S A, Carter D, Siddiqui A A. Development of a schistosomiasis vaccine. Expert Rev Vaccines. 2016; 15: 619-27. doi: 10.1586/14760584.2016.1131127.

33.- Díaz J, Florencio M. Cistitis por Schistosoma haematobium en un inmigrante subsahariano. Rev Diagn Biol 2001; 50: 45-8.

34.- Donate Moreno N J, Pastor Navarro H, Giménez Bachs J M, Carrión López P, Segura San Martín M, Salinas Sánchez A S, et al. Esquistosomiasis vesical, aportación de un caso y revisión de la literatura española. Actas Urol Esp 2006; 30: 714-9.

35.- Da Silva I M, Thiengo R, Conceicao M J, Rey L, Pereira Fhilo E, Ribeiro P C. Cystoscopy in the diagnosis and follow-up of urinary schistosomiasis in brazilian soldiers returning from Mozambique, Africa. Rev Inst Med Trop S Paulo 2006; 48: 39-42.

36.- López López A I, Cao Avellaneda E, Prieto González A, Ferri Ñiguez B, Maluff Torres A, Pérez Albacete M. Esquistosomiasis: una 
parasitosis urinaria cada vez más frecuente. Actas Urol Esp 2007; 31: 915-8.

37.- Tzanetou K, Adamis G, Andipa E, Zorzos C, Ntoumas K, Armenis K, et al. Urinary tract Schistosoma haematobium infection: a case report. J Travel Med 2007; 14: 334-7.

38.- Da Silva I M, Pereira Fhilo E, Thiengo R, Ribeiro PC, Conceicao MJ, Panasco M, et al. Schistosomiasis haematobia; histopathological course determined by cystoscopy in patient in whom praziquantel treatment failed. Rev Inst Med Trop S Paulo 2008; 50: 343-6.
39.- Álvarez Maestro M, Ríos González E, Domínguez García P, Vallejo Herrador J, Diez Rodríguez J, Martínez-Piñeiro L. Esquistosomiasis vesical: a propósito de un caso y revisión de la literatura. Ach Esp Urol 2010; 63: 554-8.

40.- Francolugo-Vélez V A, Zarzosa-Alguiar J. Infección del tracto urinario por Schistosoma haematobium. Un caso en Cuernavaca. Morelos. México. Rev Mex Urol 2010; 70 : 187-92.

41.- Sánchez-Molina M I, Sanz M P, Vicente B,
Undabeitia E, Jareño M S. Infección por Schistosoma haematobium. Semergen 2010; 36 : 529-32.

42.- García Pérez N, Arístegui Fernández J, Garrote Llanos E, Sánchez Lafuente J. Esquistosomiasis vesical: un nuevo caso importado. An Pediatr (Barc) 2014; 80 (3): e88-9.

43.- Chávez Troya $\mathrm{O}$, Molina Ramírez B, Rosales Orihuela J. Vejiga de porcelana como consecuencia de esquistosomiasis urinaria. Presentación de un caso clínico con enfoque multidisciplinario. Rev Finlay 2017; 7: 62-7. 